\title{
Micro Electromagnetic Actuator - Static Behavior
}

\author{
Constantin Daniel Comeaga ${ }^{1}$, Dragos Ovezea ${ }^{2}$ and Cristinel $l i e^{2}$ \\ ${ }^{1}$ Univ. Politehnica Bucharest, Mechatronics and Precision Eng. Department, 060042 Str. Splaiul Independentei no. 313 Bucharest, Romania \\ ${ }^{2}$ National Institute for Research\&Development in Electrical Engineering ICPE-CA, 13827850 Str. Splaiul Unirii no. 313. Bucharest, Romania
}

\begin{abstract}
The article presents a comparative study regarding the design and experimental results for a miniature electromagnetic actuator with a modified design comparing with the classical type, including a mobile array of micromagnets and a fixed coil in two configurations (planar spiral multi-layered and cylindrical). Previous work on design, modeling and simulation of this type of actuator indicated the optimum design and conducted to dimensions and material parameters. Different types of actuators were produced and experimentally tested, showing good results but also some drawbacks. Three of these design solutions are presented together with the static voltage-deflection and electrical impedances curves, experimentally derived. The results confirmed the feasibility of two actuating solution, showing good linearity and possibility to control the position at $\mathrm{h}$ accuracy and indicated improving directions.
\end{abstract}

\section{Introduction}

Micro-electromechanical systems are involved in many applications [1]. Many actuating principles were previously proposed but the highest value of the ratio between the actuating energy-volume of actuator is provided by the electromagnetic actuation, with different design solutions [2]. The electrodynamic interaction is produced between magnetic materials and electric current carrying coils and is preferred for its strength, polarity and range of displacements at micro-scale [3]. These properties recommend them for laser technology used in systems for measurements, sensors, micro-scanners, micro-mirrors with adjustable focalization and correctable aberrations, etc. $[4,5]$.

This paper is about a new type of magnetic cantilever electrodynamic actuators, with mobile array of magnets.

The common design use a fixed magnet and one or more mobile coils (cylindrical or planar).

The proposed design is provided with a fixed coil and a moving micro-magnets array and the actuation is achieved through the electrodynamic interaction between the permanent micro-magnets array and the electric current in the coils. The actual design advantages were discussed in previous papers [6,7], deduced based on modeling and simulation.

A scaled mathematical model, used in designing the studied actuators was derived [8].

Three designs, with different coil configurations and magnets, were manufactured and tested, the results being discussed in this article and showing good performances for two concepts and a poor behavior for another one. A discussion regarding possible improvements and a causal analysis of the design with poor behavior is included.

\section{The electromagnetic actuator in MEMS}

The electro-dynamic or electromagnetic (EM) actuators have complex structures and complicated driving electronics but can develop high forces at any scale and can be used at resonance or far from it.

The electromagnetic actuator type is based on the tendency of magnetic circuits to attain to the minimum potential magnetic energy or the interaction force between a magnetic field and a current-carrying wire [9].

The classical electro-dynamic actuators are similar to voice-coil actuators, widely used at macro-scale but also at miniature-scale, and based on the interaction between a fixed magnetic field and an electric current existing in a mobile coil, usually cylindrically shaped but possible to have, for the goal of miniaturization, planar shape. This design was proposed decades ago and is still used. The supply of electric current to a mobile coils rise problems at miniature scale.

This design was preferred for a long time due to the low performances of magnets (density of stored magnetic energy), imposing use of large magnets, usually cylindrically shaped, for acquiring high inductance magnetic field. Due to their high density and volume these low cost magnets, with have high mass, are difficult to be used as a mobile element for high frequency/fast response applications.

Another common solution is using a mobile part made of a high magnetic permeability material placed in the magnetic circuits obtained with the coil (as in bulk variable-reluctance electromagnets, like relays). This design is difficult to miniaturize because not only the mobile part but also the fixed part carrying the coils 
should be made of this material in order to have a good magnetic circuit. Variable reluctance actuators have a simple and robust structure, and they are widely used as a low cost switching actuators in many applications.

However, they have not gained widespread acceptance as proportional actuators due to their inherent nonlinear control characteristics. Recent improvements in the control strategies allow their use for positioning actuators but require advanced DSP controllers.

The actual improvements in the magnets behavior, having strong magnetization, allow use of mobile magnets, placed on a mobile element, and interacting with the magnetic field produced by electric currents flowing through fixed coils, as proposed in previous papers $[4,5]$.

This paper is continuing the work regarding a new type of electromagnetic actuators (EA) that may replace conventional low-frequency scanners or precision positioning actuators [4,5]. The EA is provided with a fixed coil and a moving magnets array.

The magnets array, with magnetization oriented on the actuator movement direction, was initially chosen because previous studies on bulk film magnet technology available shown defects and non-uniform magnetic parameters.

The manufacturing of the array structure impose new technological problems (a laser LIGA technology and electrodeposition were used) but removes the magnetic non-uniformity problems (even if a percentage of about $10 \%$ of the magnets volume is sacrificed for gaps, the overall performances are improved).

A few designs of cantilever structure were proposed before, with one or two leaf springs, fixed at one side of the mobile part or fixed at two opposite sides.

The structure with two springs has some advantages in the dynamical control, due to higher resonance frequencies, and the structure with opposite springs is necessary for actuators with pure translational movement, as in some optical scanners, even a strong increase of stiffness is expected.

The command was pure static for this study and only the displacement of the actuator end was considered; so the design with one leaf spring was adopted.

Three designs were studied:

- Planar multi-layer coils,

- Cylindrical coil with ferrite core,

- Cylindrical coil with empty core.

The design with cylindrical coil is used for higher electro-magnetic fields and correspondingly higher displacements. The cylindrical coil is not replacing the planar coil but added to the PCB board, with the main goal to have a double control: high displacements with the cylindrical coil and fine tuning of position with planar coil. The planar coil, obtained using the PCB technology, has 4 layers with parameters: 7 turns on each layer, turn thickness $0.035 \mathrm{~mm}$, turn width $0.3 \mathrm{~mm}$, distance between turns $0.12 \mathrm{~mm}$, cooper as material.

A description of the designs, including the dimensions and an exploded view are included in Fig. 1.

The cylindrical coil parameters are: number of turns $=$ 181 , wire diameter $\mathrm{d}=0.1 \mathrm{~mm}$, material $\mathrm{Cu} /$ doubleenamel coating $\varnothing 0.25 \mathrm{~mm}$.
The space between the magnets $(\mathrm{NdFeB})$ and the $\mathrm{PCB}$ board is $0.9 \mathrm{~mm}$ and the total thickness of PCB is $0.8 \mathrm{~mm}$.

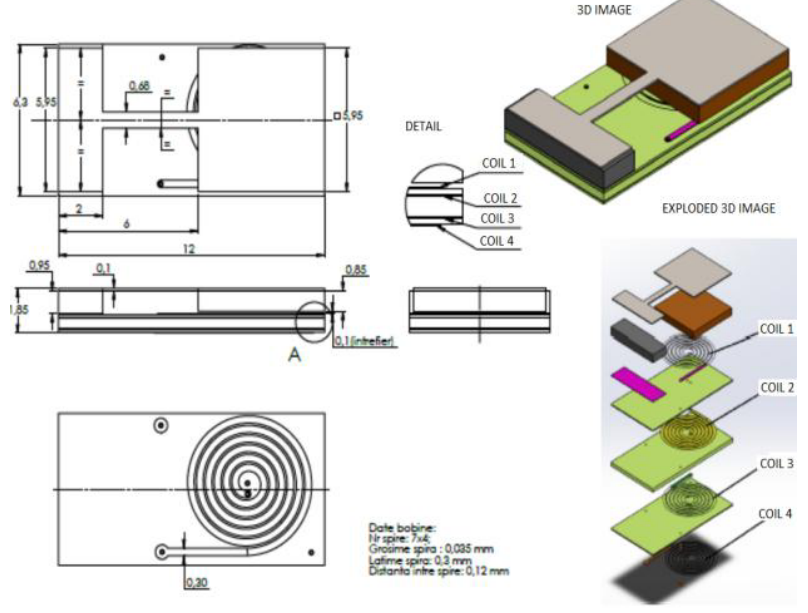

a) Actuator with planar coil,

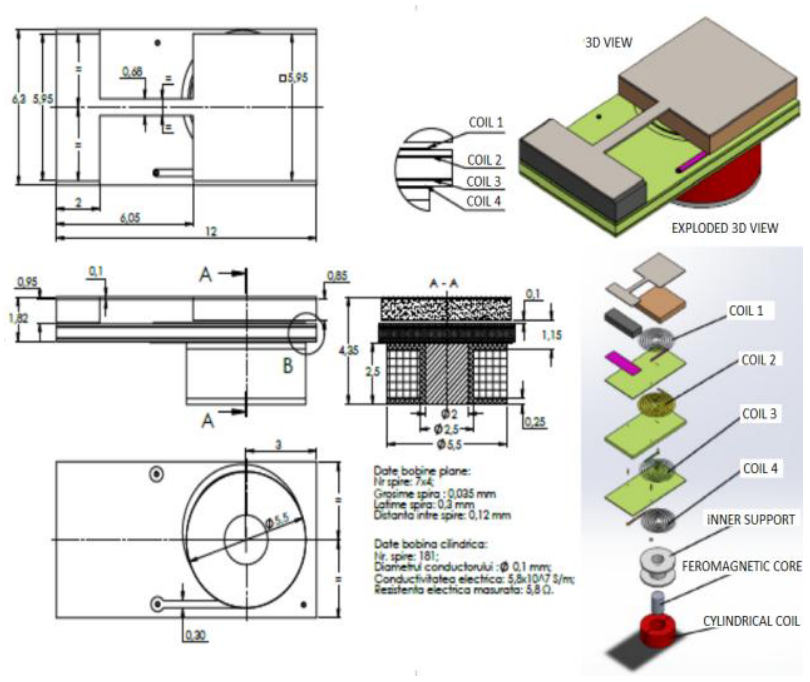

b) Actuator with cylindrical coil and fero-magnetic core
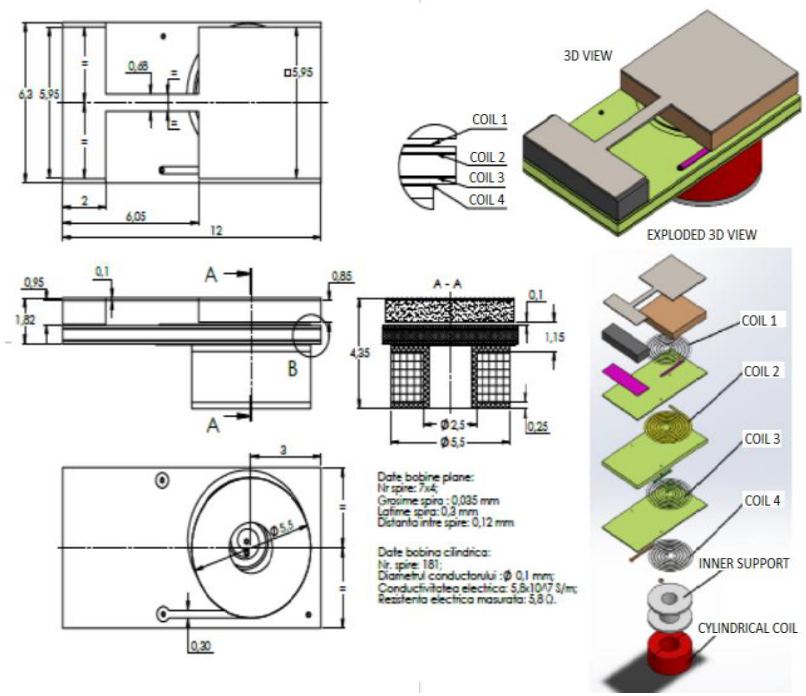

c) Actuator with cylindrical coil and without feromagnetic core

Figure 1. The tested actuators with different coils and sizes. 


\section{Experimental method and setup}

The testing was done in static regime and consisted in applying a constant electric current to the actuator, measuring the voltage drop and the displacement of the free end. The electric current was supplied by a Rhode \& Schwartz HMP4030 power source.

The voltage-current characteristic was determined in order to check for any eventual electric non-linearity in quasi-static regime. The current-displacement characteristic is defining for the behavior of the actuator.

Measurement of the free-end displacement was achieved using a white light Mirau interferometer, type WYKO NT 1100, made by Veeco. WYKO NT 1100 is a high precision interferometric microscope with a Mirau interferometer, able to scan surfaces on OZ axis, with a resolution up to $0.2 \mathrm{~nm}$, using a technique of numerical processing of the interference fringes formed by a precisely controlled changes in the position of the focal plane of the optical system along the optical axis.

The system acquires a set of interference fringes images, for various positions on $\mathrm{OZ}$ axis, which led to a matrix of values representing the estimated height of the observed surface points. The heights are evaluated with respect to a virtual center of image in order to achieve the best graphical view. The points form a mesh which models the surface features of the observed object, allowing for the extraction of various information. By operator intervention in the data processing, information such as height difference between adjacent surfaces, thickness and surface roughness can be obtained.

In the case of the measured actuator, it was necessary to be mounted on a rigid support together with a calibrated height gauge, with the role of fixed reference surface. An image of the interferometric measuring system is shown in Fig. 2.

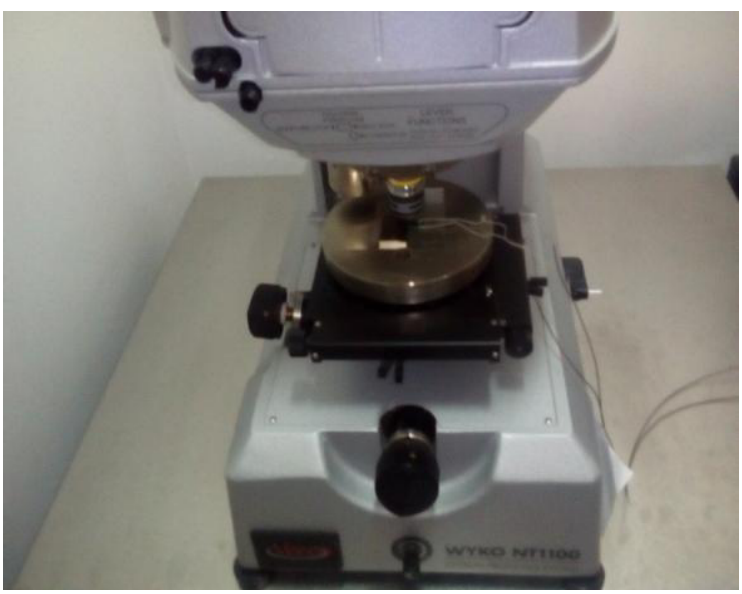

Figure 2. The experimental setup.

Fig. 3 a) shows a picture (during measurement) of the WYKO NT1100's software interface: color coded height map and processing controls in the left monitor, cameraobserved image in the right monitor and following to the right - the power supply.

Fig. $3 \mathrm{~b}$ ) shows a screen capture of the camera view (reference surface to the left, actuator end to the right and space b) with displacement data in the background.
A selection of the software interface is shown in Fig. 3 c) where the color coded surface data matrix is shown together with an $\mathrm{X}$-axis profile section.

Averaged data zones are shown in yellow. The relative height difference between the two zones is the observed displacement (taking into account the start offset, of course).

The control current was obtained by using the power source in current limiting mode. The maximum used current was set so that it would yield a current density limited to $5 \mathrm{~A} / \mathrm{mm} 2$, specific to copper conductor.

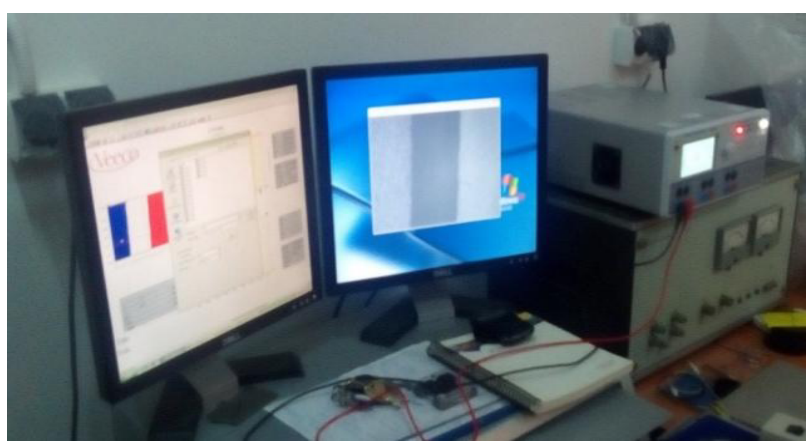

a) The control and data acquisition system

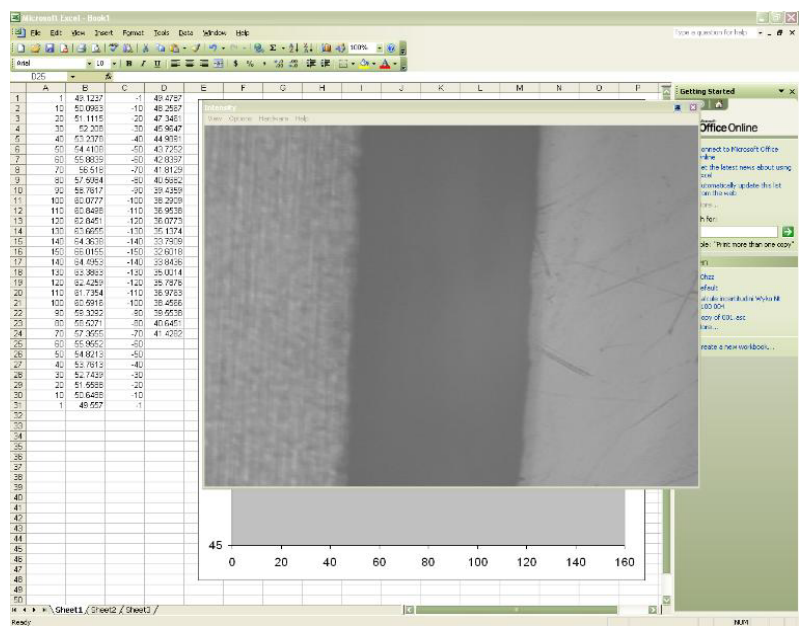

b) Actuator surface and the fixed reference surface,



c) Position profiles obtained for actuator surface and reference surface.

Figure 3. The data and image acquisition and processing. 


\section{Experimental results}

\subsection{The actuator with planar coil}

The experimental results for the actuator with planar coil and array of magnets are presented in Fig. 4.

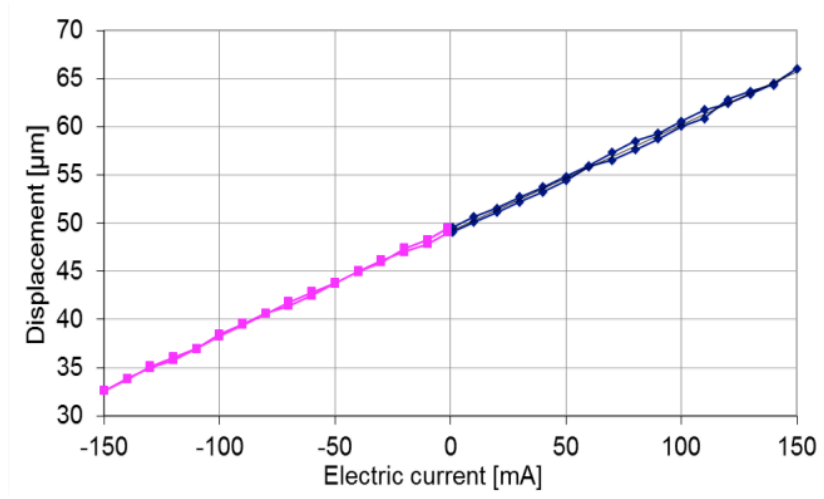

a) Characteristic electric current vs. displacement,

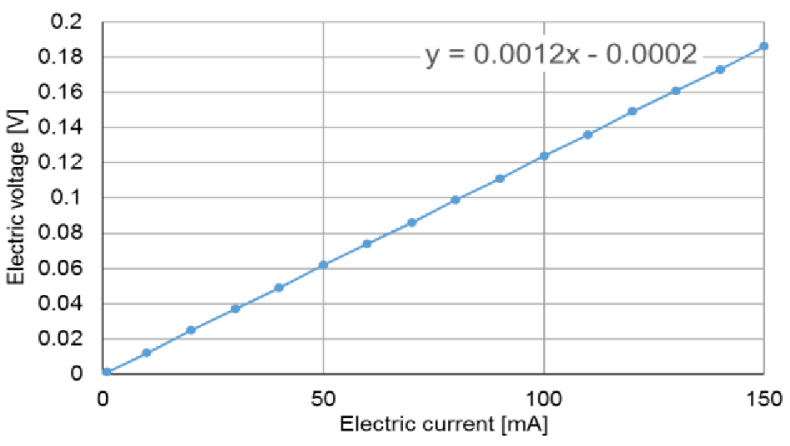

b) Characteristic electric current vs. electric voltage.

Figure 4. The experimental results for planar coils.

The electro-mechanical graph is almost linear for positive electric current and showing a small nonlinearity for negative electric currents. The useful range for long term use, due to poor heat evacuation of multi-layer structure, is limited by a $\pm 40 \mathrm{~mA}$ variation of electric current, the curve being almost linear in this range with a slope of $1.0 \mu \mathrm{m} / 10 \mathrm{~mA}$. The linearity is a strong advantage for control but the maximum displacement is limited to $\pm 4 \mu \mathrm{m}$, enough for application in nanotechnology, genetic engineering, etc.

It is possible to obtain a maximum displacement of $10-15 \mu \mathrm{m}$ for short term positioning or with the possibility of placing the actuator in a low temperature environment, extending the application range.

An increase in displacement can be obtained by:

- Increasing the current, requesting raise of thickness (limited by the standard thickness of circuit boards) or turns width (producing a growth of actuator width for a given number of turns),

- Raising the length. The ratio between length and thickness is imposed by the allowed rotation of the free end $\theta$, supposing the maximum stress omax equal with the material's limit (1). In these hypothesis, the free end displacement w could be raised only raising length (1).

$$
\theta_{\max }=\frac{\sigma_{\max }}{Y} \frac{l}{h}=\frac{3}{2} \frac{w}{l} ; w=\frac{2 \cdot \sigma_{\max }}{3 \cdot Y} \frac{l^{2}}{h}=\frac{2}{3} \cdot \theta_{\max } \cdot l
$$

where Y- Young's modulus, l-length, h-thickness. Both options are limited by the total dimensions.

\subsection{The actuator with cylindrical coil and core}

Fig. 5 presents the experimental results for the actuator with cylindrical coil, core made of ARMCO steel and using an array of magnets.

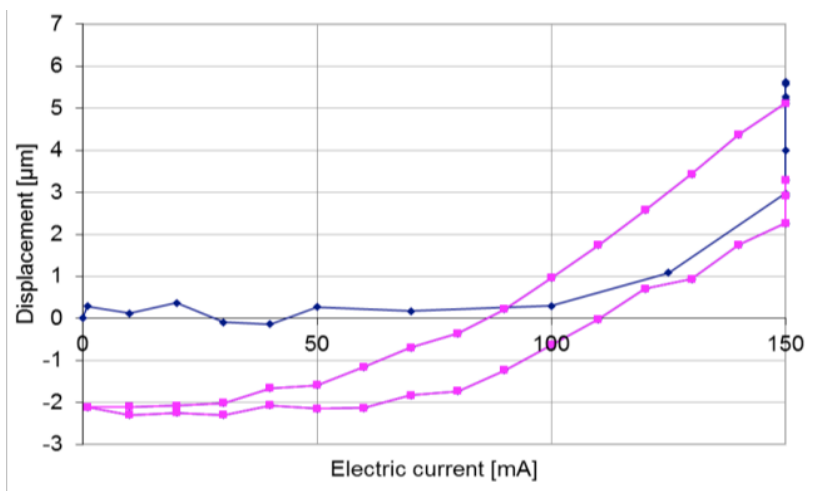

Figure 5. The experimental results for cylindrical coil with core - characteristic electric current vs. electric voltage.

An uncommon behavior could be seen here.

Using different tests and simulations it was possible to find an explanation:

- in the absence of the electric current through the coil, the magnets interact with the core and cause its magnetization; a force is acting on the mobile magnet, closing the gap between the magnets and the PCB board at the end of the mobile part;

- when the electric current is applied, if the direction of the electric current is chosen correctly, a repulsion force will act on the magnets (for opposite direction of the current, the contact between magnets and PCB is obtained on all the surface and a $-2 \mu \mathrm{m}$ displacement of the array center is measured). The magnets start to move only when this force is greater than the attracting force (small movements of the actuator orientation are measurable before detaching). If the current is higher than a value dependent on the magnetization, the magnets start to move and the equilibrium position is established when the electro-dynamic linear repulsive force is equal to the magnetic attraction non-linear force, resulting from the interaction between magnets and core; as a consequence, the curve electric current - position is not linear, and is not possible to establish a control;

- if the electric current increases close to attain the limit of maximum current density and remains constant, a slow shift of the magnet's position is observed as the core changes its magnetization state under the influence of the coil's field;

- reducing the current produces a non-linear curve, with a negative position at null current due to a different orientation of actuator as the core magnetization is removed;

- when the current is varied from zero to maximum value (negative electric current only block firmly the 
actuator), due to the equilibrium established between a linear and a non-linear varying forces, the electromechanical curve is not linear, showing high hysteresis due to the core material and the tendency for magnetization-demagnetization (the curves depend on the process speed). This kind of actuator could be used as a bi-stabile but not as a positioning actuator.

A version without core was also tested, removing the influence of the magnetization of the actuator's core.

\subsection{The actuator with cylindrical coil and without core}

The experimental results for the actuator with cylindrical coil and without core are presented in Fig. 6.

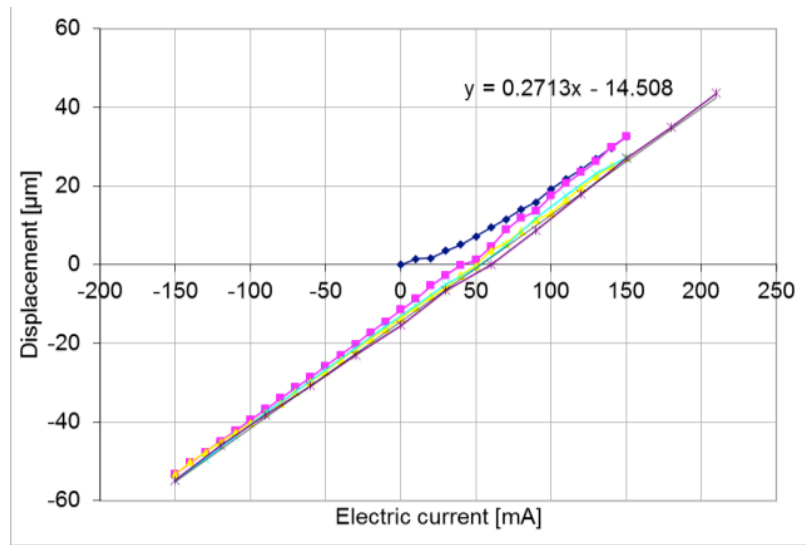

a) Characteristic electric current vs. displacement

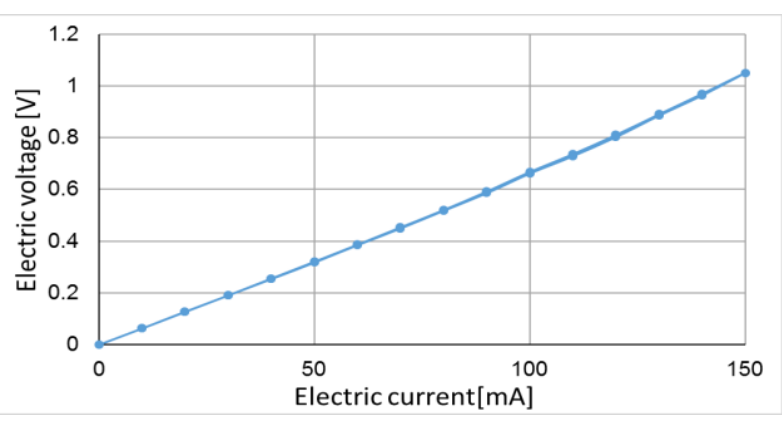

b) Characteristic electric current vs. electric voltage

Figure 6. The experimental results for indrical coil without core.

The electro-mechanical curve shows a prime nonlinear variation, quickly replaced by a linear behavior. The resting position corresponding to a null current is $14.5 \mu \mathrm{m}$ and is maintained for unlimited control variation, not influenced by the electric current variation amplitude.

If the electric current is null for a long period, the behavior is repeated, with the non-linear initial curve and subsequent linear curves. This behavior is suppose to be due to the interaction of magnet material with the electrodynamic magnetic field. The null-electric current position is stable and is included in the control algorithm.

The application range of this actuator is extended but the control should include a correction for zero. The system is heavier and bulkier, due to the cylindrical coil.

\section{Conclusions}

Three types of electromagnetic actuators with fixed coil and moving magnets were studied.

The most compact, having planar spiral coils manufactured using the technology specific to layered PCB, have a linear electro-mechanical curve without any drift or initial stabilizing position. The displacements are low, with a slope of electro-mechanical curve of $0.1 \mu \mathrm{m} / 1 \mathrm{~mA}$ and maximum displacement $4 \mu \mathrm{m}$ for long term use. An improved version uses a miniature cylindrical coil, obtaining a slope of electro-mechanical curve of $0.27 \mu \mathrm{m} / 1 \mathrm{~mA}$ and maximum $10 \mu \mathrm{m}$ for long term use, producing a repeatable displacement of zero-current position.

Another design, using a coil core, proved unuseful.

The planar coils proved to be unseful and of comparable performance with cylindrical coils if the overall volume is considered, requiring optimization studies, improvements in manufacturing technologies to help heat removal and thicker layers of cooper. More studies regarding the coils optimum dimensions and number of turns should be made in the future, including the efficiency of a combined solution with planar and cylindrical coils, for combined actuation or the possibility to use one for actuation and another for sensing.

\section{Acknolwdgment}

The work was conducted in the framework of AEEL PNCD II PT PCCA 249/2014 research grant, code PN-IIPT-PCCA-2013-4-1557, financed by the Romanian National Authority for R\&D and Innovation - UEFISCDI.

\section{References}

1. N.P. Mahalik, MEMS (Tata McGraw-Hill Publ. Company, 2007).

2. M. Gad-el-Hak, The MEMS Hanbook 2nd Ed. (CRC Press, 2005).

3. O. Cougat, J. Delamare, and G. Reyne, IEEE Trans. on Magnetics, 39, 3607-3612 (2003).

4. J. Yan, S. Luanava, and V. Casasanta, Proc. SPIE, 4985, 115-120 (2003).

5. L. K. Lagorce, O. Brand, M.G. Allen, IEEE J. Microelectromech. Syst., 8, 2-9 (1999).

6. A.M. Morega, N. Tanase, N., M. Popa, M. Morega, J.B. Dumitru, Proc. of the 8th Int. Symposium on Advanced Topics in Electrical Engineering, DOI: 10.1109/ATEE.2013.6563478 (2013).

7. A.M. Morega, N. Tanase, M. Morega, C.D. Comeaga C. Ilie, Proc. of the 9th International Symposium on Advanced Topics in Electrical Engineering (ATEE), DOI: 10.1109/ATEE.2015.7133869 (2015).

8. A.M. Morega, M. Morega, C.D. Comeaga, A. Dobre, Proc. of the 9th International Symposium on Advanced Topics in Electrical Engineering (ATEE), DOI: 10.1109/ICATE.2016.7754662, (2016).

9. E. Purcell, Electricity and Magnetism, 2nd ed., (Cambridge Univesity Press, 2011). 